Commun. Korean Math. Soc. 27 (2012), No. 3, pp. 489-496

http://dx.doi.org/10.4134/CKMS.2012.27.3.489

\title{
SMARANDACHE WEAK BE-ALGEBRAS
}

\author{
Arsham Borumand Saeid
}

ABstract. In this paper, we introduce the notions of Smarandache weak $B E$-algebra, $Q$-Smarandache filters and $Q$-Smarandache ideals. We show that a nonempty subset $F$ of a $B E$-algebra $X$ is a $Q$-Smarandache filter if and only if $A(x, y) \subseteq F$, which $A(x, y)$ is a $Q$-Smarandache upper set The relationship between these notions are stated and proved.

\section{Introduction}

The Smarandache algebraic structures theory was introduced in 1998 by R. Padilla [9]. In [5], Kandasamy studied of Smarandache groupoids, subgroupoids, ideal of groupoids, seminormal sub-groupoids, Smarandache Bol groupoids, and strong Bol groupoids and obtained many interesting results about them. Smarandache semigroups are very important for the study of congruences, and they were studied by Padilla [9].

A Smarandache weak structure on a set $S$ means a structure on $S$ that has a proper subset $P$ with a weaker structure. By proper subset of a set $S$, we mean a subset $P$ of $S$, different from the empty set, from the original set $S$, and from the idempotent elements if any.

In [4], Borumand Saeid et al. studied the concept of Smarandache $B C H$ algebras and obtained many interesting results about Smarandache (fresh, clean and fantastic) ideal in a $B C H$-algebras. Smarandache $B L$-algebras have been invented by Borumand Saeid et al. [3], and they dealed with Smarandache ideal structures in Smarandache $B L$-algebras.

Recently, H. S. Kim and Y. H. Kim defined a BE-algebra [6]. S. S. Ahn and K. S. So defined the notion of ideals in $B E$-algebras, and then stated and proved several characterizations of such ideals [2]. In [8], B. L. Meng introduced the notion of an $C I$-algebra as a generalization of a $B E$-algebra.

Received July 5, 2011; Revised November 17, 2011.

2010 Mathematics Subject Classification. Primary 06F35, 03G25.

$K e y$ words and phrases. $C I$-algebras, BE-algebra, Smarandache weak BE-algebra, $(Q$ Smarandache) filter, ( $Q$-Smarandache) ideal. 


\section{Preliminaries}

Definition $2.1([6])$. An algebra $(X ; *, 1)$ of type $(2,0)$ is called a $B E$-algebra if

(BE1) $x * x=1$

(BE2) $x * 1=1$;

(BE3) $1 * x=x$;

(BE4) $x *(y * z)=y *(x * z)$,

for all $x, y, z \in X$.

We introduce a relation " $\leq$ " on $X$ by $x \leq y$ if and only if $x * y=1$.

Proposition $2.2([6])$. If $(X ; *, 1)$ is a BE-algebra, then $x *(y * x)=1$ for any $x, y \in X$.

Definition $2.3([8])$. An algebra $(X ; *, 1)$ of type $(2,0)$ is called a $C I$-algebra if

(CI1) $x * x=1$;

(CI2) $1 * x=x$;

(CI3) $x *(y * z)=y *(x * z)$,

for all $x, y, z \in X$.

Definition $2.4([6])$. Let $(X, *, 1)$ be a $B E$-algebra and $F$ be a non-empty subset of $X$. Then $F$ is said to be a filter of $X$ if

(F1) $1 \in F$;

(F2) $x * y \in F$ and $x \in F$ imply $y \in F$.

Definition 2.5 ([1]). A non-empty subset $I$ of a $B E$-algebra $X$ is called an ideal of $X$ if it satisfies:

(I1) $x \in X$ and $a \in I$ imply $x * a \in I$, i.e., $X * I \subseteq I$;

(I2) $x \in X, a, b \in I$ imply $(a *(b * x)) * x \in I$.

Proposition 2.6 ([1]). Let $I$ be an ideal of a BE-algebra $X$. If $a \in I$ and $a \leq x$, then $x \in I$.

Definition $2.7([7])$. Let $X$ be a $C I$-algebra and $x, y \in X$. Define $A(x, y)$ by

$$
A(x, y):=\{z \in X: x *(y * z)=1\} .
$$

We call $A(x, y)$ an upper set of $x$ and $y$.

Definition 2.8 ([7]). A $C I$-algebra or a $B E$-algebra $X$ is said to be self distributive if $x *(y * z)=(x * y) *(x * z)$ for all $x, y, z \in X$.

Proposition 2.9 ([10]). Let $X$ be a self distributive BE-algebra. Then for all $x, y, z \in X$ the following statements hold:

(1) if $x \leq y$, then $z * x \leq z * y$;

(2) $y * z \leq(x * y) *(x * z)$; 


\section{Smarandache weak $B E$-algebras}

Note that every $B E$-algebra is a $C I$-algebra, but the converse is not true. A $C I$-algebra which is not a $B E$-algebra is called a proper $C I$-algebra.

Definition 3.1. A Smarandache weak $B E$-algebra $X$ is said to be a $B E$ algebra $X$ in which there exists a proper subset $Q$ of $X$ such that

(S1) $1 \in Q$ and $|Q| \geqslant 2$;

(S2) $Q$ is a $C I$-algebra under the operation of $X$.

Example 3.2. Let $X:=\{1, a, b, c\}$ be a set with the following table:

\begin{tabular}{c|cccc}
$*$ & 1 & $a$ & $b$ & $c$ \\
\hline 1 & 1 & $a$ & $b$ & $c$ \\
$a$ & 1 & 1 & $a$ & $a$ \\
$b$ & 1 & 1 & 1 & 1 \\
$c$ & 1 & 1 & 1 & 1
\end{tabular}

Then $(X, *, 1)$ is a $B E$-algebra. If $Q$ is one of the sets $\{1, a\},\{1, b\},\{1, c\}$, $\{1, a, b\}$ or $\{1, a, c\}$, then $Q$ is a $C I$-algebra. So $X$ is a Smarandache weak $B E$-algebra.

Example 3.3. Let $X:=\{1, a, b, c\}$ be a set with the following table:

\begin{tabular}{c|cccc}
$*$ & 1 & $a$ & $b$ & $c$ \\
\hline 1 & 1 & $a$ & $b$ & $c$ \\
$a$ & 1 & 1 & $a$ & $a$ \\
$b$ & 1 & 1 & 1 & $a$ \\
$c$ & 1 & 1 & 1 & 1
\end{tabular}

Then it is a $B E$-algebra, but $Q=\{1, b, c\}$ is not a $C I$-algebra and $X$ is not a Smarandache weak $B E$-algebra.

Definition 3.4. A nonempty subset $F$ of a $B E$-algebra $X$ is called a Smarandache filter of $X$ related to $Q$ (or briefly, $Q$-Smarandache filter of $X$ ) if it satisfies:

(SF1) $1 \in F$;

(SF2) $(\forall y \in Q)(\forall x \in F)(x * y \in F \Rightarrow y \in F)$.

Example 3.5. In Example 3.2, if $Q=\{1, a\}$, then $F=\{1, b\}$ is a $Q$ Smarandache filter of $X$, but $F=\{1, a\}$ is not a Smarandache filter of $X$.

Note. If $F$ is a Smarandache filter of a $B E$-algebra $X$ related to every $C I$ algebra contained in $X$, we simply say that $F$ is a Smarandache filter of $X$.

Proposition 3.6. If $\left\{F_{\lambda}: \lambda \in \Delta\right\}$ is an indexed set of $Q$-Smarandache filters of a BE-algebra $X$, where $\Delta \neq \varnothing$, then $F=\cap\left\{F_{\lambda}: \lambda \in \Lambda\right\}$ is a $Q$-Smarandache filter of $X$.

Proposition 3.7. Any filter $F$ of a BE-algebra $X$ is a $Q$-Smarandache filter of $X$. 
Note. By the following example we show that the converse of above proposition is not correct in general.

Example 3.8. Let $X:=\{1, a, b, c\}$ be a set with the following table:

\begin{tabular}{l|llll}
$*$ & 1 & $a$ & $b$ & $c$ \\
\hline 1 & 1 & $a$ & $b$ & $c$ \\
$a$ & 1 & 1 & $b$ & $c$ \\
$b$ & 1 & $a$ & 1 & $c$ \\
$c$ & $c$ & $c$ & $c$ & 1
\end{tabular}

Then $X$ is a $B E$-algebra and $Q=\{1, b\}$ is a $C I$-algebra which is properly contained in $B E$-algebra $X$ and $F=\{1, b\}$ is a $Q$-Smarandache filter of $X$ but it is not a filter of $X$ because $b * a \in F$ and $b \in F$, but $a \notin F$.

Proposition 3.9. If $F$ is a $Q$-Smarandache filter $F$ of a self distributive BEalgebra $X$, then $(\forall x, y, z \in Q)(z *(y * x) \in F, z * y \in F \Rightarrow z * x \in F)$.

Proof. Since $z *(y * x)=(z * y) *(z * x) \in F$ and $z * y \in F$, by (SF2) we have $z * x \in F$.

Proposition 3.10. If $F$ is a $Q$-Smarandache filter of a self distributive BEalgebra $X$, then $(\forall x, y \in Q)(y *(y * x) \in F \Rightarrow y * x \in F)$.

Proof. Assume that $y *(y * x) \in F$ for all $x, y \in Q$ since $y * y=1 \in F$, by (SF1) and Proposition 3.9 we have $y * x \in F$.

Proposition 3.11. Let $F$ be a $Q$-Smarandache filter of a BE-algebra $X$. Then

(1) $F \neq \varnothing$.

(2) If $x \in F, x \leq y, y \in Q$, then $y \in F$.

(3) If $X$ is a self distributive CI-algebra and $x, y \in F$, then $x * y \in F$.

Proof. (1) Since $F$ is a $Q$-Smarandache filter of $X$, therefore by (SF1) we have $1 \in F$. Hence $F \neq \emptyset$.

(2) Let $x \in F, x \leq y$ and $y \in Q$. Then $x * y=1 \in F$, therefore by (SF2) we get that $y \in F$.

(3) We have $y *(x *(x * y))=x *(y *(x * y))=x *(x * 1)=x * 1=1$, and hence $y *(x *(x * y)) \in F$. Since $y \in F$, by (SF2) $x *(x * y) \in F$. It follows from Proposition $3.10 x * y \in F$.

Proposition 3.12. If $F$ is a $Q$-Smarandache filter of a BE-algebra $X$ and if $Q$ satisfies $X * Q \subseteq Q$, then $(\forall x, y \in F)(\forall z \in Q)(x *(y * z)=1 \Rightarrow z \in F)$.

Proof. Assume that $X * Q \subseteq Q$. Let $F$ be a $Q$-Smarandache filter of $X$. Suppose that $x *(y * z)=1$ where $x, y \in F$ and $z \in Q$. Then $y * z \in Q$ by the hypothesis and $x *(y * z) \in F$ we have $y * z \in F$. By (SF2), $z \in F$. This completes the proof. 
Theorem 3.13. Let $Q_{1}$ and $Q_{2}$ be $C I$-algebras which are properly contained in a BE-algebra $X$ and $Q_{1} \subseteq Q_{2}$. Then every $Q_{2}$-Smarandache filter is a $Q_{1}$-Smarandache filter of $X$.

Note. By the following example we show that the converse of above theorem is not correct in general.

Example 3.14. In Example 3.8, $Q_{1}=\{1, b\}, Q_{2}=\{1, b, c\}$ are $C I$-algebras which are properly contained in $X$. It is easily checked that $F=\{1, b\}$ is a $Q_{1}$-Smarandache filter of $X$ and is not a $Q_{2}$-Smarandache filter of $X$, since $c * a=c \in F$, but $a \notin F$.

Proposition 3.15. Let $X$ be a self distributive BE-algebra and $F$ be a $Q$ Smarandache filter of $X$. Then $F_{a}=\{x: a * x \in F\}$ is a $Q$-Smarandache filter, for any $a \in X$.

Proof. Since $a * a=1 \in F, a \in F_{a}$ and so $\varnothing \neq F_{a}$. Assume $x * y \in F_{a}$ and $x \in F_{a}$. Then $a *(x * y) \in F$ and $a * x \in F$. By self distributivity we have $(a * x) *(a * y) \in F$ and $a * x \in F$. Thus $a * y \in F$ and so $y \in F_{a}$. Therefore $F_{a}$ is a $Q$-Smarandache filter of $X$.

Definition 3.16. Let $X$ be a $B E$-algebra and $x, y \in X, Q \subset X$ be a $C I$ algebra. Define

$$
A(x, y):=\{z \in Q: x *(y * z)=1\} .
$$

We call $A(x, y)$ a $Q$-Smarandache upper set of $x$ and $y$.

Note. It is easy to see that $1, x, y \in A(x, y)$. The set $A(x, y)$, where $x, y \in Q$, need not be a $Q$-Smarandache filter of $X$ in general. In Example 3.2, it is easy to check that $A(1, a)=\{a\}$, which means that $A(1, a)$ is not a $Q$-Smarandache filter of $X$.

Proposition 3.17. Let $X$ be a self distributive BE-algebra. Then the $Q$ Smarandache upper set $A(x, y)$ is a $Q$-Smarandache filter of $X$, where $x, y \in Q$.

Proof. Since $x * 1=1$, for all $x \in Q, 1 \in A(x, y)$. Let $a * b \in A(x, y)$ and $a \in A(x, y)$, where $b \in Q$. Then $1=x *(y *(a * b))$ and $1=x *(y * a)$. It follows from the definition of the self distributivity

$$
\begin{aligned}
1 & =x *(y *(a * b))=x *((y * a) *(y * b)) \\
& =(x *(y * a)) *(x *(y * b))=1 *(x *(y * b))=x *(y * b) .
\end{aligned}
$$

Therefore $b \in A(x, y)$. This proves that $A(x, y)$ is a $Q$-Smarandache filter of $X$.

Theorem 3.18. If $F$ is a non-empty subset of a BE-algebra $X$, then $F$ is a $Q$-Smarandache filter of $X$ if and only if $A(x, y) \subseteq F$ for all $x, y \in F$.

Proof. Assume that $F$ is a $Q$-Smarandache filter of $X$ and $x, y \in F$. If $z \in$ $A(x, y)$, then $x *(y * z)=1 \in F$. Then by Proposition 3.12, $z \in F$. Hence $A(x, y) \subseteq F$. 
Conversely, suppose that $A(x, y) \subseteq F$ for all $x, y \in F$. Since $x *(y * 1)=x * 1=1$, $1 \in A(x, y) \subseteq F$. Assume $a * b, a \in F$. Since $(a * b) *(a * b)=1$, we have $b \in A(a * b, a) \subseteq F$. Hence $F$ is a $Q$-Smarandache filter of $X$.

Theorem 3.19. If $F$ is a $Q$-Smarandache filter of a BE-algebra $X$, then

$$
F=\cup_{x, y \in F} A(x, y) \text {. }
$$

Proof. Let $F$ be a $Q$-Smarandache filter of $X$ and $z \in F$. Since $z *(1 * z)=$ $z * z=1$, we have $z \in A(z, 1)$. Hence

$$
F \subseteq \cup_{z \in F} A(z, 1) \subseteq \cup_{x, y \in F} A(x, y) .
$$

If $z \in \cup_{x, y \in F} A(x, y)$, then there exist $a, b \in F$ such that $z \in A(a, b)$. It follows from Theorem 3.18 that $z \in F$. This means that $\cup_{x, y \in F} A(x, y) \subseteq F$.

Theorem 3.20. If $F$ is a $Q$-Smarandache filter of BE-algebra $X$, then

$$
F=\cup_{x \in F} A(x, 1) .
$$

Proof. Let $F$ be a $Q$-Smarandache filter of $X$ and $z \in F$. Since $z *(1 * z)=$ $z * z=1$, we have $z \in A(z, 1)$. Hence

$$
F \subseteq \cup_{z \in F} A(z, 1) .
$$

If $z \in \cup_{x \in F} A(x, 1)$, then there exists $a \in F$ such that $z \in A(a, 1)$, which means that $a * z=a *(1 * z)=1 \in F$. Since $F$ is a $Q$-Smarandache filter of $X$ and $a \in F$, we have $z \in F$. This means that $\cup_{x \in F} A(x, 1) \subseteq F$.

Definition 3.21. A nonempty subset $I$ of a $B E$-algebra $X$ is called a Smarandache ideal of $X$ related to $Q$ (or briefly, a $Q$-Smarandache ideal of $X$ ) if it satisfies:

(SI1) $x \in Q$ and $a \in I$ imply $x * a \in I$, i.e., $Q * I \subseteq I$

(SI2) $(\forall x \in Q)(\forall a, b \in I)$ imply $(a *(b * x)) * x \in I$.

Example 3.22. In Example 3.2, $Q=\{1, a\}$ is a $C I$-algebra of $X$ and $I=$ $\{1, a, b\}$ is a Smarandache ideal of $Q$, but $J=\{1, c\}$ is not a $Q$-Smarandache ideal of $X$ because $c, 1 \in J$ and $a \in Q$, but $(c *(1 * a)) * a=(c * a) * a=1 * a=$ $a \notin J$.

Lemma 3.23. Let $X$ be a CI-algebra. Then

(1) Every $Q$-Smarandache ideal I of $X$ contains 1 ;

(2) If $I$ is a $Q$-Smarandache ideal of $X$, then $(a * x) * x \in I$ for all $a \in I$ and $x \in Q$.

Proof. (1) Let $\varnothing \neq I$ be a $Q$-Smarandache ideal of $X$. For $x \in I, 1=x * x \in$ $I * I \subseteq Q * I \subseteq I$. Thus $1 \in I$.

(2) Let $b:=1$ in (SI2). Then $(a *(1 * x)) * x \in I$. Hence $(a * x) * x \in I$.

Lemma 3.24. A nonempty subset $I$ of a BE-algebra $X$ is a $Q$-Smarandache ideal of $X$ if and only if it satisfies 
(1) $1 \in I$;

(2) $(\forall x, z \in Q)(\forall y \in I)(x *(y * z) \in I \Rightarrow x * z \in I)$.

Theorem 3.25. Let $Q_{1}$ and $Q_{2}$ be $C I$-algebras which are properly contained in a BE-algebra $X$ and $Q_{1} \subseteq Q_{2}$. Then every $Q_{2}$-Smarandache ideal of $X$ is a $Q_{1}$-Smarandache ideal.

\section{Conclusion}

In this paper, we have introduced the concept of Smarandache weak $B E$ algebras and investigated some of their useful properties. It is well known that the ideals and filters with special properties play an important role in the logic system. The aim of this article is to investigate Smarandache ideals and Smarandache filters in $B E$-algebras.

We believe that these results are very useful in developing algebraic structures. Also these definitions and main results can be similarly extended to some other algebraic systems such as lattices and Lie algebras.

In our future study of Smarandache structures of $B E$-algebras, the following topics should be considered:

(1) To get more results in Smarandache weak BE-algebras and its applications;

(2) To get more connections between $B E$-algebras and Smarandache weak $B E$-algebras;

(3) To define another Smarandache structures;

(4) To define a fuzzy structure of Smarandache (weak) $B E$-algebras.

Acknowledgments. The author is highly grateful to referees for their valuable comments and suggestions which were helpful in improving this paper.

\section{References}

[1] S. S. Ahn and K. S. So, On generalized upper sets in BE-algebras, Bull. Korean Math. Soc. 46 (2009), no. 2, 281-287.

[2] _ On ideals and upper sets in BE-algebras, Sci. Math. Jpn. 68 (2008), no. 2, 279-285.

[3] A. Borumand Saeid, A. Ahadpanah, and L. Torkzadeh, Smarandache BL-algebra, J. Appl. Log. 8 (2010), 235-261.

[4] A. Borumand Saeid and A. Namdar, Smarandache BCH-algebra, World Applied Sciences Journal 7 (2009), 77-83.

[5] W. B. V. Kandasamy, Smarandache gropoids, http://www.gallup.unm.edu/ Smarandache/Groupoids.pdf.

[6] H. S. Kim and Y. H. Kim, On BE-algebras, Sci. Math. Jpn. Online e-2006 (2006), 1299-1302.

[7] H. K. Kyung, A Note on CI-algebras, Int. Math. Forum, 6 (2011), no. 1, 1-5.

[8] B. L. Meng, CI-algebra, Sci. Math. Jpn. Online e-2009 (2009), 695-701.

[9] R. Padilla, Smarandache algebraic structures, Bull. Pure Appl. Sci. Sect. E Math. Stat. 17E (1998), no. 1, 119-121.

[10] A. Rezaei and A. Borumand Saeid, Some results in BE-algebras, Analele Universitatii Oradea Fasc. Matematica, Tom XIX (2012), 33-44. 
Department of Mathematics

Shahid Bahonar University of Kerman

KERMAN 7616914111, IRAN

E-mail address: arsham@mail.uk.ac.ir 